\title{
The Effect of Quail Egg and Hen Egg Consumption on Low-Density Lipoprotein Oxidation and Small Dense Low-Density Lipoprotein
}

\author{
Raveenan Mingpakanee, Ph.D. ${ }^{1}$, Chatchanok Chaisitthichai, Bs.C. ${ }^{2}$, \\ Nattaporn Wichitamporn, Bs.C. ${ }^{2}$, Paradee Sappittayakorn, Bs.C. ${ }^{2}$, \\ Suparnnikar Phongphanwatana, Bs.C. ${ }^{2}$
}

${ }^{1}$ Department of Transfusion Medicine and Clinical Microbiology, ${ }^{2}$ Medical Technology Program, Faculty of Allied Health Sciences, Chulalongkorn University, Pathumwan, Bangkok 10330, Thailand.

Received 27 September 2018 • Revised 19 February 2019 • Accepted 4 March 2019 • Published online 28 March 2019

\section{Abstract:}

Objective: The aim of the study was to investigate the effect of quail egg and hen egg supplements on lipoprotein profiles, low-density lipoprotein (LDL) oxidation and small dense LDL cholesterol (sd-LDL-C) in young healthy people, compared with hen eggs.

Material and Methods: Twenty-three healthy volunteers (11 men and 12 women) were randomly assigned to consume 3 whole hen eggs per day (hen group, $n=11$ ) (total cholesterol $633 \mathrm{mg}$ ) or 9 quail eggs per day (quail group, $n=12$ ) (total cholesterol $459 \mathrm{mg}$ ) for 30 days. The plasma cholesterol and plasma triglyceride concentrations and lipoprotein fractions (Triglyceride-rich lipoprotein; TRL, LDL and high-density lipoprotein; HDL) were determined at baseline and after the 30-day period of egg consumption. The LDL oxidation (lag time) was measured by the increase of conjugated diene production. Sd-LDL-C was calculated from the major lipid and lipoprotein parameters.

Results: In the quail group, plasma triglyceride (TG) and LDL-TG were significantly decreased, whereas the plasma cholesterol and HDL-C were unchanged. There was no alteration in lipoprotein profiles in the hen group. The LDL lag time of the quail group was longer than at baseline. There were no significant changes in sd-LDL-C levels in both groups during the study.

Conclusion: Quail egg and hen egg consumptions for 30 days did not change the lipoprotein profiles, sd-LDL as well as the LDL-oxidation, which not modified the cardiovascular disease risk factor.

Keywords: hen egg, LDL-oxidation, lipoprotein profiles, quail egg, small dense LDL

Contact: Raveenan Mingpakanee, Ph.D.

Department of Transfusion Medicine and Clinical Microbiology

Faculty of Allied Health Sciences, Chulalongkorn University, Pathumwan,

Bangkok 10330, Thailand.

E-mail: mraveenan@gmail.com, raveenan.m@chula.ac.th
J Health Sci Med Res 2019;37(2):109-120 doi: 10.31584 /jhsmr.201946 www.jhsmr.org 


\section{Introduction}

Cardiovascular disease (CVD) is a major cause of death in Thailand. ${ }^{1}$ The major risk factor for CVD is dyslipidemia which is defined by hypercholesterolemia, high levels of low-density lipoprotein cholesterol (LDL-C) and low levels of high-density lipoprotein cholesterol (HDL-C). ${ }^{2}$ Low-density lipoprotein (LDL) particles are heterogeneous in size and density, the LDL diameter varies between 18-25 nm., and density varies between 1.019-1.063 g/ml. ${ }^{3}$ LDL particles were classified into subclasses, mainly large, buoyant LDL (Ib-LDL) and small dense LDL particles (sd-LDL). The sd-LDL is a strong risk factor for CVD., ${ }^{4,5}$ The origin of CVD is the LDL particles, can penetrate into arterial wall and undergo oxidation to become oxidized LDL (ox-LDL). The ox-LDL is taken up by macrophage via scavenger receptor. Macrophage can uptake ox-LDL continuously and become lipid laden macrophages, accumulating in the arterial wall. ${ }^{6}$ The evidences supported the relationship between ox-LDL and the pathogenesis of atherosclerosis are; the ox-LDL was found in the atherosclerotic lesion, LDL extracted from atherosclerotic lesion has a biological property of ox-LDL, and the antibodies to ox-LDL presented in the circulation. Then, the LDLoxidation can be used as atherosclerotic risk factor. ${ }^{7}$ Another important parameter that closely related to CVD is sd-LDL. In a case control study reported by Austin et al. $^{4}$, LDL mean particle diameters isolated from myocardial infarction patients were smaller than control LDL. Sd-LDL was associated with a threefold increase risk of myocardial infarction independent of age, sex and body weight. Serum cholesterol and LDL-C of case and control were not significantly different. Then, the use of plasma cholesterol and LDL-C to estimate risk for CVD could not be precisely predicted.

Eggs are enriched in dietary cholesterol and many nutrients, such as essential amino acids, folate, riboflavin, selenium, choline, lutein, zeaxanthin, vitamin A, D, E, and
$\mathrm{K}$ and fatty acids. ${ }^{8}$ Due to the eggs contained high cholesterol, regular egg consumption may also affect plasma cholesterol and LDL-C. The American Heart Association/American College of Cardiology reported that there is insufficient evidence to support the restriction of dietary cholesterol, which is less relevant to CVD. ${ }^{9}$ Many researchers have tried to elucidate the relationship between egg consumption and plasma lipid and lipoprotein i.e. plasma cholesterol and LDL-C. Some reports have shown the egg consumption improved the lipoprotein profiles $^{10,11}$, others were increased the plasma lipid. ${ }^{12}$ However, there has been little evidence that egg consumption is directly related to CVD. One research confirmed the 2 hen egg consumption per day, up to 6 weeks, egg did not adversely affect endothelial function which was measured as flow-mediated vasodilation and brachial artery reactivity in healthy adults and arterial stuffiness or endothelial function. ${ }^{13}$ Many studies reported the effects of hen egg consumption on the alteration of lipoproteins profiles, but few studies reported the alteration of LDL oxidation and sd-LDL-C. One hen egg per day for 4 weeks can decrease the LDL-oxidation, which reported by Kishimoto et $\mathrm{al}^{14}$, whereas Mutungi et al. $^{15}$ reported the sd-LDL-C was decreased after 3 hen eggs per day consumption for 12 weeks.

Egg yolks from different species are difference in lipid and nutrients. The fatty acid profile form of 5 avian species, hen, quail, goose, turkey and ostrich, were studied by Adabi et al. ${ }^{16}$ The ostrich egg had the highest unsaturated fatty acid and the lowest cholesterol content in relation to the other avian species. Hen yolk contained higher levels of 22:6n-3 than those found in other avian species. Compared with a hen egg, the cholesterol contained in a quail egg is $11.12 \mathrm{mg} / \mathrm{g}$ of yolk, whereas the hen egg contains $12.25 \mathrm{mg} / \mathrm{g}$ of yolk. From this report, the cholesterol concentration in the hen egg is higher than that in the quail egg. Sinanoglou et al. ${ }^{17}$ analyzed the 
major lipid compositions in the egg yolk of 5 avian species: quail, goose, turkey, duck and ostrich. The turkey and goose egg yolks contained a beneficial $\omega-6 / \omega-3$ ratio contributing to cardiovascular risk reduction. The quail egg yolk showed a significantly low concentration in total lipid, triglycerides, cholesterol and saturated fatty acids. The researchers recommended that the quail egg is suitable for a healthier human and especially infant diet. In Thailand, the most popular egg is the hen egg, but the quail egg is a composition of many kinds of food. The chemical and lipid compositions in egg yolk vary within the species, quail eggs contain lower cholesterol and triglycerides, and thus it may be an alternative egg choice for food. Most research reported the effect of egg consumption on plasma lipid and lipoproteins, however, the serum cholesterol and LDL-C levels are not able to predict the risk for CVD accurately. The LDL-oxidation and sd-LDL-C should be added to the CVD risk evaluation. No published research elucidates the change in blood lipid and lipoprotein profile after quail egg consumption, as well as the sd-LDL and LDL-oxidation. Therefore, this study was undertaken to evaluate the effects of quail egg and hen egg consumption on lipoprotein profile, LDL-oxidation and sd-LDL-C in young healthy volunteers.

\section{Material and Methods}

\section{Subjects}

Twenty-four volunteers aged between 18-25 years (12 male and 12 female) were recruited. The inclusion criteria were normolipidemia; fasting plasma cholesterol less than $220 \mathrm{mg} / \mathrm{dl}$, plasma triglycerides less than 150 $\mathrm{mg} / \mathrm{dl}$. Participants were excluded if they had egg allergy, diabetes mellitus, liver or kidney disorder, body mass index (BMI) higher than $22.9 \mathrm{~kg} / \mathrm{m}^{2}$. All volunteers were randomly assigned to consume 3 hen eggs (50 g/ unit) per day (hen group) or 9 quail eggs (10 g/unit) per day (quail group) in their regular diets for 30 days.
Hen eggs and quail eggs were cooked by boiling. Hen eggs and quail eggs were purchased at a local supermarket (Top supermarket). Participants were asked not to consume any eggs outside of those provided to them. Participants were instructed to maintain their regular dietary habits, medication, and physical activity and asked to avoid other egg consumption. The 3 hen eggs (50 g/egg) contained cholesterol $642 \mathrm{mg}, 11.7 \mathrm{~g}$ fat, $4.4 \mathrm{~g}$ SFA, $4.8 \mathrm{~g}$ MUFA, $2.4 \mathrm{~g}$ PUFA. The 9 quail egg contained cholesterol $459 \mathrm{mg} \mathrm{kcal}, 13.2 \mathrm{~g}$ fat, $4.6 \mathrm{~g}$ SFA, $6.3 \mathrm{~g}$ MUFA, $2.9 \mathrm{~g}$ PUFA. ${ }^{18}$ Written informed consent was obtained from the subjects after an explanation of the purpose, nature and potential risks of this study. This study was approved by the Ethics Committee of Health Sciences, Chulalongkorn University. The Certificate of Approval number is 076/ 2017.

\section{Body weight}

Body weight was measured by balance-type medical scale with light clothing and without shoes. Percentage of body fat was measured using Bioelectric Impedance Analysis. All parameters were measured at the beginning for baseline and after the 30-day period of quail or hen egg consumption. BMI was calculated by body weight divided by height in meters. ${ }^{2}$

\section{Blood collection}

Twelve-hour fasting blood sample was collected from all the volunteers before and after the 30-day period of hen or quail egg consumption using ethylenediamine tetra aceitc acid (EDTA) as an anticoagulant. The blood was centrifuged at 3,000xg, for $10 \mathrm{~min}$, EDTA plasma was separated immediately after centrifugation.

\section{Lipoprotein separations $^{19}$}

Lipoproteins were isolated from EDTA plasma by sequential ultracentrifugation. Triglyceride-rich lipoprotein 
(TRL), LDL and HDL were isolated at a density range of $1.006-1.019 \mathrm{~g} / \mathrm{ml}, 1.019-1.063 \mathrm{~g} / \mathrm{ml}$ and $1.063-1.21$ $\mathrm{g} / \mathrm{ml}$, respectively. Ultracentrifugation was performed by himac CS150GXL Microultracentrifuge (Hitachi KoKi, Tokyo, Japan). Total cholesterol (TC) and triglyceride (TG) concentrations in each lipoprotein fraction were determined by enzymatic methods (Human, Germany).

\section{Susceptibility of low-density lipoprotein to} oxidation, in vitro

LDL was desalted with phosphate buffered saline (PBS) (pH 7.4) by gel filtration in Sephadex G-25M PD-10 columns (Amersham, USA), using PBS $(0.1 \mathrm{~mol} / \mathrm{L})$ free of EDTA. The protein concentration in the LDL fraction was measured by the Lowry method ${ }^{20}$, using bovine serum albumin as the standard. LDL (50 $\mu \mathrm{g}$ protein $/ \mathrm{ml})$ was incubated at $37^{\circ} \mathrm{C}$ in the presence of $5 \mu \mathrm{M} \mathrm{CuSO}_{4}$ in PBS buffer. The formation of conjugated diene was monitored by continuously increasing absorption at $234 \mathrm{~nm}$ for up to 4 hours (UV-Visible spectrophotometer Evolution 300 (Thermo Scientific, USA). The lag time for initiation of LDL oxidation was defined as the time interval between the initiation and the intercept of the two tangents draw to the lag and propagation phase of the absorbance curve at $234 \mathrm{~nm}^{21}$

\section{Small dense low-density lipoprotein cholesterol concentration $^{22}$}

Sd-LDL-C concentration was estimated using the formula: sd-LDL-C (mg/dl)=0.58(non-HDL-C)+0.407 (direct LDL-C)-0.719(calculated LDL-C)-12.05. Direct LDL-C were measured by homogenous assay, performed by Professional Laboratory Management Corp. Co., Ltd. Bangkok, Thailand. LDL was calculated using the Firedewald equation. ${ }^{23}$

\section{Statistical analysis}

The results are reported as means \pm standard deviation. Data analysis was conducted using Statistical Package for the Social Sciences (SPSS) program statistical software (ver.17.0). The significant differences of demographic data and biochemical data at baseline and after the test period between quail group and hen group were determined by Independent student's t-test. The change in lipoprotein profile and LDL lag time between baseline and after the 30-day period of egg consumption were analyzed by a Paired t-test. Two factor ANOVA was used to test for differences between the sexes. P-value< 0.05 was considered statistically significant.

\section{Results}

\section{Demographic data of participants}

Twenty-four volunteers participated in this study. One participant in the hen group dropped out because he could not consume the 3 eggs daily for 30 days. Baseline characteristics of volunteers are shown in Table 1. The mean age of participants was $19.1 \pm 2.0$ years in the quail group, and 19.6 \pm 1.5 years in the hen group. Body weight was $58.4 \pm 4.1 \mathrm{~kg}$ in the quail group, and $56.3 \pm 8.4 \mathrm{~kg}$ in the hen group. BMl was $20.4 \pm 1.4 \mathrm{~kg} / \mathrm{m}^{2}$ in the quail group, and $20.5 \pm 1.8 \mathrm{~kg} / \mathrm{m}^{2}$ in the hen group. Body fat was $18.7 \pm 6.1 \%$ in the quail group, and $19.0 \pm 6.0 \%$ in the hen group. There were no significant differences between age, body weight, BMl and \% body fat between quail and hen group. \% Body fat in females were significantly higher than males ( $p$-value<0.01). After 30 days of egg consumption, there were no significant changes in body weight, BMI and $\%$ body fat in both groups. No sex differences were noted in any of the measured parameters in response to egg. 


\section{Lipoprotein profiles}

Baseline characteristic of lipoprotein profiles are in Table 2. There were no significant differences in baseline lipid and lipoproteins between the 2 groups. After 30 day of quail egg consumption, the plasma TG and LDL-TG concentrations were significantly lower than baseline ( $p$-value<0.05), whereas the VLDL-TG and HDL-TG levels were not significantly change. Plasma TG and LDLTG were reduced by 11.4 and $13.0 \%$, respectively. The plasma TC, TRL-C, LDL-C and HDL-C concentrations were not significantly different between baseline and after 30 days of quail egg consumption (Table 3). Plasma TG, TC and other lipoprotein profiles for the hen group did not change over the period, although participants consumed more cholesterol provided by the eggs (Table 4).
The LDL oxidation was conducted by conjugated diene monitoring. Lag time was calculated by the interception between slope and steady state. LDL isolated from the quail group shows a prolonged lag time compared to baseline $(63.5 \pm 5.3$ vs $79.5 \pm 4.7 \mathrm{~min}$ at baseline and after the 30-day period of quail egg consumption, respectively, $p$-value=0.01). In the hen group, there were no significant changes in lag time for LDL oxidation $(66.7 \pm 3.19$ vs $71.8 \pm$ 4.6 $\mathrm{min}$ at baseline and after 30 days of quail egg consumption, respectively, $\mathrm{p}-\mathrm{value}=0.14$ ) (Figure 1). SdLDL-C concentrations were estimated from lipid and lipoprotein parameters. Compared to baseline, there were no significant changes in the sd-LDL-C concentration for either group (Figure 2).

Table 1 Demographic data of participants at baseline and after 30 days of quail egg and hen egg consumption

\begin{tabular}{|c|c|c|c|c|}
\hline \multirow{2}{*}{ Parameters } & \multicolumn{2}{|c|}{ Quail group (n=12) } & \multicolumn{2}{|c|}{ Hen group $(n=11)$} \\
\hline & Baseline & 30-day & Baseline & 30-day \\
\hline \multicolumn{5}{|l|}{ Sex } \\
\hline Male & 6 & 6 & 5 & 5 \\
\hline Female & 6 & 6 & 6 & 6 \\
\hline Age (year) & $19.1 \pm 2.0$ & $19.1 \pm 2.0$ & $19.6 \pm 1.5$ & $19.6 \pm 1.5$ \\
\hline Body weight (kg) & $58.4 \pm 4.1$ & $59.1 \pm 4.4$ & $56.3 \pm 8.4$ & $56.0 \pm 8.2$ \\
\hline Male & $59.1 \pm 3.7$ & $58.4 \pm 3.5$ & $59.1 \pm 8.5$ & $59.1 \pm 8.5$ \\
\hline Female & $57.8 \pm 4.2$ & $59.8 \pm 5.8$ & $53.6 \pm 7.7$ & $53.5 \pm 7.8$ \\
\hline Body mass index $\left(\mathrm{kg} / \mathrm{m}^{2}\right)$ & $20.4 \pm 1.4$ & $20.6 \pm 1.3$ & $20.5 \pm 1.8$ & $20.9 \pm 1.3$ \\
\hline Male & $20.1 \pm 1.0$ & $19.9 \pm 0.8$ & $20.5 \pm 1.7$ & $20.3 \pm 1.6$ \\
\hline Female & $20.6 \pm 1.8$ & $21.3 \pm 1.4$ & $20.6 \pm 2.2$ & $20.5 \pm 2.1$ \\
\hline Body fat (\%) & $18.7 \pm 6.1$ & $18.4 \pm 6.5$ & $19.0 \pm 6.0$ & $18.5 \pm 5.7$ \\
\hline Male & $14.2 \pm 2.8$ & $13.9 \pm 3.2$ & $14.7 \pm 2.8$ & $14.2 \pm 2.8$ \\
\hline Female & $23.2 \pm 5.0^{*}$ & $22.9 \pm 5.8^{*}$ & $22.6 \pm 5.7^{*}$ & $22.1 \pm 5.0^{*}$ \\
\hline
\end{tabular}

Data are shown in mean \pm S.D. There were no differences between baseline and 30 -day in both groups.

*Significantly different from male at $p$-value $<0.05$. 
Table 2 Baseline characteristic of lipoprotein profiles of the participants

\begin{tabular}{lll}
\hline Lipoprotein profiles & Quail group $(\mathbf{n = 1 2})$ & Hen group $(\mathbf{n = 1 1})$ \\
\hline Plasma cholesterol (mg/dl) & $181.4 \pm 26.7$ & $189.8 \pm 25.4$ \\
TRL-C & $30.5 \pm 18.5$ & $32.1 \pm 7.8$ \\
LDL-C & $74.2 \pm 14.4$ & $76.8 \pm 11.4$ \\
HDL-C & $60.4 \pm 10.7$ & $60.8 \pm 12.7$ \\
Plasma triglyceride (mg/dl) & $91.1 \pm 22.8$ & $77.0 \pm 19.1$ \\
TRL-TG & $45.6 \pm 21.4$ & $34.7 \pm 10.7$ \\
LDL-TG & $24.8 \pm 6.1$ & $20.0 \pm 4.5$ \\
HDL-TG & $23.5 \pm 2.8$ & $20.8 \pm 4.2$ \\
sd-LDL-C (mg/dl) & $29.4 \pm 7.1$ & $31.0 \pm 6.2$ \\
LDL lag time (min) & $64.8 \pm 17.0$ & $66.8 \pm 10.6$ \\
\hline
\end{tabular}

Data are shown in mean \pm S.D. There were no differences between baseline and 30-day in both groups.

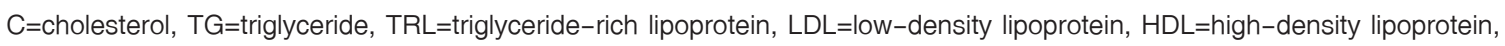
sd-LDL=small dense low-density lipoprotein

Table 3 Lipoprotein profiles of the quail group

\begin{tabular}{lllll}
\hline Lipoprotein profiles & Baseline & 30-day & Change & P-value \\
\hline Plasma C (mg/dl) & $181.4 \pm 26.7$ & $189.4 \pm 23.4$ & +8.0 & 0.25 \\
TRL-C & $30.5 \pm 18.5$ & $27.4 \pm 14.7$ & -3.1 & 0.63 \\
LDL-C & $74.2 \pm 14.4$ & $75.8 \pm 19.3$ & +1.6 & 0.75 \\
HDL-C & $60.4 \pm 10.7$ & $64.6 \pm 23.4$ & +4.2 & 0.54 \\
Plasma TG $(\mathrm{mg} / \mathrm{dl})$ & $91.1 \pm 22.8$ & $80.7 \pm 21.2^{*}$ & -10.4 & 0.03 \\
TRL-TG & $45.6 \pm 21.4$ & $39.7 \pm 17.5$ & -5.9 & 0.20 \\
LDL-TG & $24.8 \pm 6.1$ & $18.1 \pm 6.8^{*}$ & -6.7 & 0.005 \\
HDL-TG & $23.5 \pm 2.8$ & $20.9 \pm 7.4$ & -2.6 & 0.24 \\
\hline
\end{tabular}

Data are shown in mean $\pm S D$. *Significantly different from baseline at $p$-value $<0.05$.

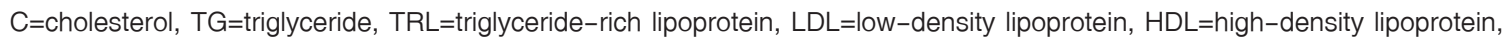
sd-LDL=small dense low-density lipoprotein 
Table 4 Lipoprotein profiles of the hen group

\begin{tabular}{|c|c|c|c|c|}
\hline Lipoprotein profiles & Baseline & 30-day & Change & P-value \\
\hline Plasma C (mg/dl) & $189.8 \pm 25.4$ & $184.9 \pm 26.5$ & -4.9 & 0.51 \\
\hline TRL-C & $32.1 \pm 13.1$ & $27.6 \pm 12.3$ & -4.5 & 0.27 \\
\hline LDL-C & $76.8 \pm 13.0$ & $72.9 \pm 19.3$ & -3.9 & 0.45 \\
\hline $\mathrm{HDL}-\mathrm{C}$ & $60.8 \pm 12.7$ & $57.4 \pm 16.9$ & -2.1 & 0.47 \\
\hline Plasma TG (mg/dl) & $77.0 \pm 19.1$ & $77.4 \pm 18.4$ & +0.4 & 0.95 \\
\hline TRL-TG & $34.7 \pm 10.7$ & $36.2 \pm 14.1$ & +1.5 & 0.77 \\
\hline LDL-TG & $20.0 \pm 4.5$ & $18.4 \pm 4.2$ & -1.6 & 0.34 \\
\hline HDL-TG & $20.8 \pm 4.2$ & $19.7 \pm 7.0$ & -1.0 & 0.33 \\
\hline
\end{tabular}

Data are shown in mean \pm S.D. *Significantly different from baseline at $p$-value $<0.05$.

$\mathrm{C}=$ cholesterol, $\mathrm{TG}=$ triglyceride, $\mathrm{TRL}=$ triglyceride-rich lipoprotein, $\mathrm{LDL}=$ low-density lipoprotein, $\mathrm{HDL}=$ high-density lipoprotein, sd-LDL=small dense low-density lipoprotein

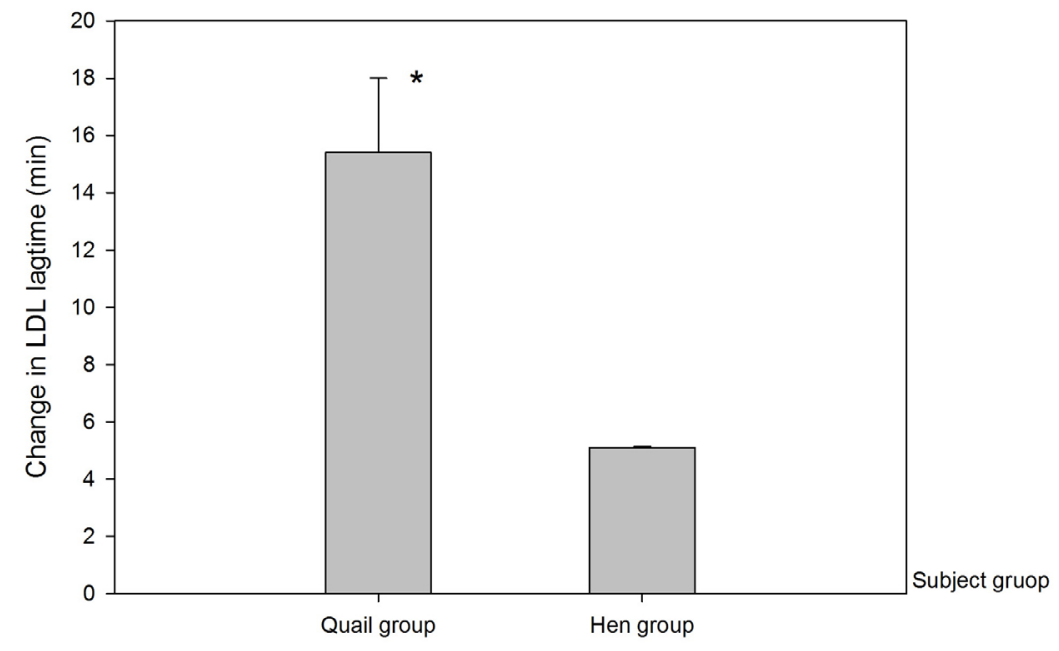

*Indicates significantly different from baseline at $p$-value $<0.05$.

Figure 1 The changes in low-density lipoprotein lag time in quail group and hen group. Values ware expressed as mean changes between baseline and after 30 days of quail and egg consumption. 


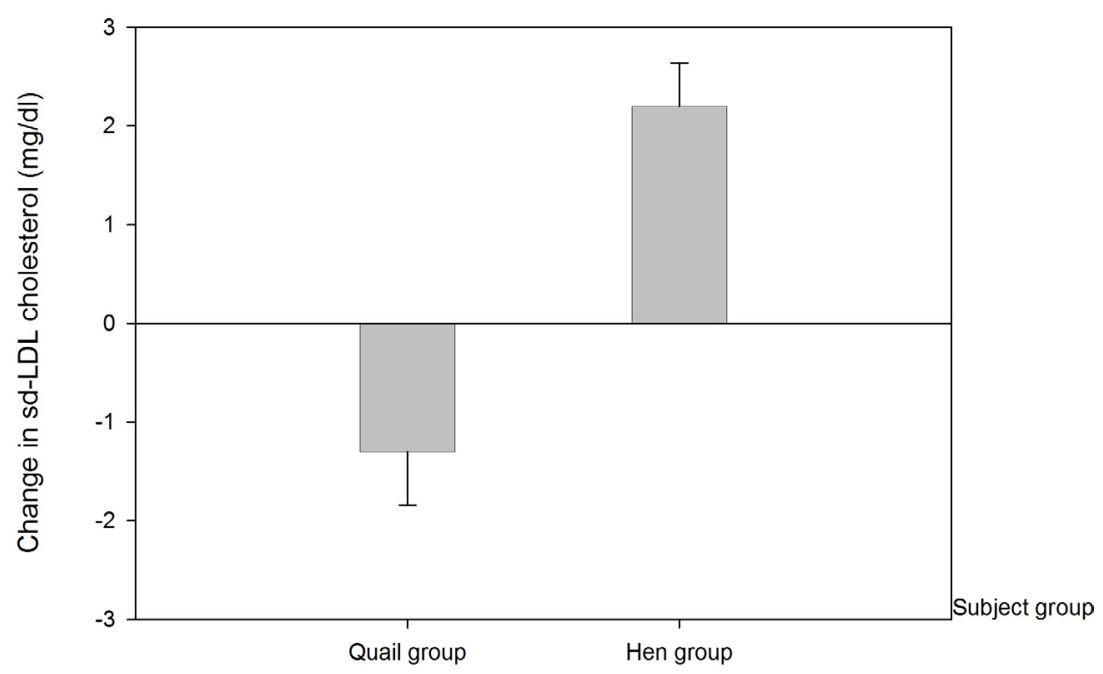

Figure 2 The changes in small, dense low-density lipoprotein cholesterol concentration from baseline and after 30 days of quail egg (quail group) and hen egg (hen group) consumption. Values ware expressed as mean changes between baseline and 30-day. There were no differences between baseline and 30-day in both groups.

\section{Discussion}

In this study, we demonstrated that quail egg supplement significantly decreases the plasma TG and LDL-TG without changing the plasma TC, LDL-C and sdLDL-C concentrations. Furthermore, quail egg intake prolong the lag time for LDL oxidation. The hen egg consumption did not increase lipoprotein profiles. The LDL-oxidation and sd-LDL-C did not modified after 30 day daily 3 -hen egg consumption.

There are very few researches studying the effect of quail egg consumption on plasma lipid and lipoproteins. One research that studied the plasma lipid changes after quail egg consumption was reported by Adabi et al. ${ }^{16}$ Four male participants were consumed $21 \mathrm{~g}$ quail egg yolks per day (approximate 7 quail eggs) without diet restricted, for 30 days. At the end of the study, the LDL-C was significantly increased, compared with baseline. Plasma cholesterol and triglyceride, as well as, HDL-C did not change during the study. The difference between the current study and Adabi's study may due to the age of participants. The average age of participants in the current study was 19 years old while the Adabi's study was 55 years old. The age-related raise the plasma cholesterol and LDL-C. ${ }^{24}$ This may be explained by the effect of age on LDL-C metabolism. LDL particles are removed from the circulation by hepatic LDL receptor, and the LDL receptor decreases with age, then reducing the rate of LDL clearance and increase the LDL resident time in the circulation. ${ }^{25}$ Older volunteers have reduced hepatic LDL receptor formation, resulting in less LDL clearance from the bloodstream, resulting in a high LDL-C. Moreover, the reduction of plasma TG and LDL-TG was found in current study. Since the quail group lost more weight when compared with hen group, the weight loss increases the lipoprotein lipase activity which would facilitate TG hydrolysis. ${ }^{26}$ It is conceivable that the decrease in plasma TG in the present study may results from the weight reduction. 
The effects of hen egg supplements on plasma lipid and lipoproteins also investigated. Our results represent that daily hen egg consumption did not increase plasma TC, plasma TG and LDL-C, in young healthy volunteers. This finding is different from a previous study. Oh and Miller studied the supplement of 3 eggs/day in healthy middle-aged men, in addition to daily diets for 30 days. After 30 days, the plasma TC was increased from $188.0 \pm 36.0$ to $199.0 \pm 36.0 \mathrm{mg} / \mathrm{dll}^{27}$ Other research reported the effects of 2 hen egg consumption for 3 weeks increased the plasma TC and LDL-C in middle-aged men. ${ }^{12}$ The difference in age of participants between the current study and previous studies may account to the cholesterol response. Bile acid synthesis declines with age, and then the cholesterol utilization also decrease which is accompanied by rise plasma cholesterol. The cholesterol-rich diet may increase the TC and LDL-C, and then an increasing intake of dietary cholesterol from egg, in combination with energy restriction should be recommended. The diet was restricted during egg consumption as found in some studies. Egg consumption in overweight men with a carbohydrate-restricted diet was conducted by Mutungi and colleagues (2008). ${ }^{29}$ Overweight men (BMl 25-37 kg/m²) restricted carbohydrates (10.0-15.0\% energy from carbohydrates) and supplements to 3 eggs per day, for 12 weeks. At the end of the study, the concentration of plasma TG was reduced, whereas the HDL-C was significantly increased. Plasma LDL-C and LDL-C:HDL-C ratio did not change. The similar research which study on overweight men supplemented 2 eggs per day for 12 weeks does not increase plasma TC and LDL-C. ${ }^{30}$ Although our research did not restrict carbohydrates, a 3-hen-egg supplement did not increase the TC or LDL$\mathrm{C}$, and did not modify the CVD risk factors.

Most research studying on the effects of hen egg consumption reported the plasma TC, TG, LDL-C and HDL-C concentrations, but TG content in each lipoprotein was not elucidated. Another finding on lipoprotein profiles after quail egg consumption in the current study was the reduction of LDL-TG concentrations. Normally, LDL particles are enriched in cholesteryl ester, but in some state, the LDL particles were induced to change the lipid composition and become TG-rich LDL. Human LDL particles comprise 2 different main fractions: Ib-LDL and sd-LDL particles. ${ }^{31,32}$ Lb-LDL is defined as LDL particle with a diameter $\geq 25.5 \mathrm{~nm}$, whereas $\mathrm{sd}-\mathrm{LDL}$ has a diameter smaller than $25.5 \mathrm{~nm}$. The sd-LDL production depends on the cholesteryl ester in LDL particle exchanged with TG in very low-density lipoprotein (VLDL) particles. After the exchange, the LDL particle becomes a TG-rich LDL. TG in LDL undergoes hydrolysis by lipoprotein lipase, and becomes a smaller and denser LDL, which is more atherogenic than the $\mathrm{lb}-\mathrm{LDL}{ }^{33}$ The main features of sdLDL are more atherogenic than Ib-LDL; sd-LDL particles easily penetrate into the arterial wall and are more susceptible to oxidation due to a deficiency in vitamin $\mathrm{E}^{34}$ Evidence has shown that Sd-LDL particles are an independent risk factor for the development of CVD., ${ }^{4,535}$ People with predominated sd-LDL particles have a 3-fold increased risk for developing CVD independent of age, sex, and relative weight. ${ }^{4}$ Furthermore, LDL particle were positively correlated with plasma HDL-C levels and negatively correlated with plasma TG levels. ${ }^{36}$

In the quail group, the volunteers showed significantly decreased plasma TG and LDL-TG (Table 3). According to the sd-LDL generation, reduction in $T G$ concentration in the VLDL particle leads to a decrease in the core lipid exchange between VLDL and LDL particles. In this experiment, LDL-TG concentration in the quail group was significantly decreased at the end of the study (24.8 \pm 6.1 vs $18.1 \pm 6.8 \mathrm{mg} / \mathrm{dl}$ at baseline and 30-day quail egg consumption, respectively). The sd-LDL generation also decreased. From our result, the sd-LDL-C concentration of the quail group trend to decline, not significantly 
decreased (Figure 2). The sd-LDL-C in the hen group was also calculated. After 30 days of hen egg consumption, the sd-LDL-C levels did not significantly change. The reduction of sd-LDL after 3 hen eggs consumption per day for 12 weeks was reported by Mutungi et al. and Blesso et al. ${ }^{15,37}$ However, all participants followed a carbohydrate-restricted diet during 12 weeks, and showed a decrease in plasma TG and sd-LDL which modified the CVD risk factor. Carbohydrate-restriction modified lipoprotein metabolism by reducing plasma TG, VLDL-TG and sd-LDL ${ }^{38}$, and sd-LDL levels correlated with plasma TG. ${ }^{4}$ In this study, volunteers were not restricted the carbohydrate diet, there was no change in plasma TG as well as sd-LDL at the end of study.

The important beginning mechanism for CVD is LDL oxidation. LDL particles are oxidized by reactive oxygen species within the arterial wall in order to become ox-LDL. The ox-LDL is not recognized by the LDL receptor, but it increases binding to the scavenger receptor on macrophage, leading to the development of lipid laden macrophage or foam cells. ${ }^{6}$ The method for the continuous monitoring of LDL oxidation is conjugated diene formation, in vitro. Using this method, the conjugated diene formation is monitored by an increase in absorbance at $234 \mathrm{mn}$. The LDL lag time is defined as LDL susceptibility to oxidation. Our results reveal that after a 30-day period of quail egg consumption, LDL lag time significantly increased (Figure 1). One mechanism that can be explained is the reduction of $T G$ in $L D L$ particles, which leads to a decrease in sd-LDL formation. The sd-LDL is more susceptible to oxidation than $\mathrm{Ib}-\mathrm{LDL}^{34}$; thus, the reduction of sd-LDL causes the prolonged LDL lag time. In hen group, there was no change in LDL-oxidation after 30 day of egg consumption. This finding is consistent with the prior study which 3 hen egg consumption per day did not increase the LDL oxidation. Because lutein and zeaxanthin are antioxidant enzyme and rich in egg, intake 2-3 egg per day can increase the plasma lutein and zeaxanthin concentrations. Due to the egg is a good source of lutein and zeaxanthin, then egg consumption can increase the plasma lutein and zeaxanthin which may protect the LDL-oxidation.

This is the preliminary study on the effect of quail egg consumption on lipoprotein profiles, sd-LDL and LDL-oxidation. Limitations of this study were the participants in quail group was higher plasma TG than those in the hen group, and therefore may have benefited more from a plasma triglyceride reduction. The cholesterol intakes from hen group and quail group are not comparable, therefore we were not able to compare the benefit of hen egg and quail egg consumption. Furthermore, the dietary intake did not record during the study, then it may vary in the nutrients. Further study is needed to address more participants to evaluate quail egg consumption on the lipoprotein profiles, sd-LDL as well as LDL oxidation, and study in other populations, for example, the elderly people or population with some underlying diseases, such as diabetes or CVD.

\section{Conclusion}

This study demonstrates that quail egg and hen egg consumptions for 30 days did not change the lipoprotein profiles, sd-LDL as well as the LDL-oxidation, which not modified the CVD risk factor. It may be suggest that the quail egg is an alternative egg for healthy people.

\section{Acknowledgement}

This research was supported by The Grant for the Development of New Faculty Staff, Chulalongkorn University, and the Research Fund of the Faculty of Allied Health Sciences, Chulalongkorn University. 


\section{Conflict of Interest}

The authors have no conflict of interest to declare.

\section{References}

1. Strategy and Planning Division. The number and rate of patients with ischemic heart disease per 100,000 population (2007-2015) [homepage on the Internet]. Nonthaburi: Bureau of Non Communicable Disease, Ministry of Public Health; 2016 [cited 2017 Nov 28]. Available from http://www.thaincd.com/ 2016

2. Tsioufis C, Mantzouranis E, Kalos T, Konstantinidis D, Tousoulis D. Risk factors of atherosclerosis: pathophysiological mechanisms. In: Tousoulis D, editor. Coronary artery disease, from biology to clinical practice. London: Academic Press; 2018;p.43-66.

3. Williams PT, Vranizan KM, Krauss RM. Correlations of plasma lipoproteins with LDL subfractions by particle size in men and women. J Lipid Res 1992;33:765-74.

4. Austin MA, Breslow JL, Hennekens CH, Buring JE, Willett WC, Krauss RM. Low-density lipoprotein subclass patterns and risk of myocardial infarction. JAMA 1988;260:1917-21.

5. Gardner CD, Fortmann SP, Krauss RM. Association of small low-density lipoprotein particles with the incidence of coronary artery disease in men and women. JAMA 1996;18: 276:875-81.

6. Durrington PN. Lipoprotein and their metabolism. In: Durrington $P N$, editor. Hyperlipidemia: diagnosis and management. $2^{\text {nd }}$ ed. Oxford: Butterworth Heinemann; 1995;p.25-71.

7. Seghrouchni I, Drai J, Bannier E, Garcia I, Revol A. Lowdensity lipoprotein (LDL) behavior after in vitro oxidation in three groups of diabetics. Farmaco 2001;56:471-4.

8. Song WO, Kerver JM. Nutritional contribution of eggs to American diets. J Am Coll Nutr 2000;19(5 Suppl):S556-62.

9. Stone NJ, Robinson JG, Lichtenstein AH, Bairey Merz CN, Blum CB, Eckel RH, et al. 2013 ACC/AHA guideline on the treatment of blood cholesterol to reduce atherosclerotic cardiovascular risk in adults: a report of the American College of Cardiology/American Heart Association Task Force on Practice Guidelines. Circulation 2014;129(25 Suppl 2): S76-9.

10. Djousse L, Gaziano JM. Egg consumption in relation to cardiovascular disease and mortality: the physicians' health study. Am J Clin Nutr 2008;87:964-9.

11. Fernandez ML. Dietary cholesterol provided by eggs and plasma lipoproteins in healthy populations. Curr Opin Clin Nutr 2006;9:8-16.

12. Levy Y, Maor I, Presser D, Aviram M. Cinsumption of eggs with meals increases the susceptibility of human plasma and low-density lipoprotein to lipid peroxidation. Ann Nutr Metab 1996;40:243-51.

13. Katz DL, Evans MA, Nawaz H, Njike VY, Chan W, Comerford $\mathrm{BP}$, et al. Egg consumption and endothelial function: a randomized controlled crossover trial. Int J Cardiol 2005; 99:65-70.

14. Kishimoto $Y$, Taguchi C, Saita E, Suzuki-Sugihara N, Nishiyama $\mathrm{H}$, Wang $\mathrm{W}$, et al. Additional consumption of one egg per day increases serum lutein plus zeaxanthin concentration and lowers oxidized low-density lipoprotein in moderately hypercholesterolemic males. Food Res Int 2017;99:944-9.

15. Mutungi G, Waters D, Ratliff J, Puglisi M, Clark RM, Volek JS, et al. Eggs distinctly modulate plasma carotenoid and lipoprotein subclasses in adult men following a carbohydraterestricted diet. J Nutr Biochem 2010;21:261-7.

16. Adabi SHG, Ahbab M, Fani AR, Hajbabaei A, Ceylan N, Cooper RG. Egg yolk fatty acid profile of avian speciesinfluence on human nutrition. J Anim Physiol Anim Nutri 2013; 97:27-38.

17. Sinanoglou VJ, Strati IF, Miniadis-Meimaroglou S. Lipid, fatty acid and carotenoid content of edible egg yolks from avian species: a comparative study. Food Chem 2011;124: 971-7.

18. Tanjor S, Sriwan T, Puwastien P, Deeaum A, Judprasong K. Nutritive value of commonly consumed eggs and effects of cooking. Thai Sci Technol J 2015;23:651-6.

19. Havel RJ, Eder HA, Bragdon JH. The distribution and chemical composition of ultracentrifugally separated lipoproteins in human serum. J Clin Invest 1955;34:1345-53.

20. Lowry $\mathrm{OH}$, Rosebrough NJ, Farr AL, Randall RJ. Protein measurement with the folin phenol reagent. $J$ Biol Chem 1951;193:265-75.

21. Parthasarathy S, Khoo JC, Miller E, Barnett J, Witztum JL, Steinberg D. Low density lipoprotein rich in oleic acid is protected against oxidative modification: implications for dietary prevention of atherosclerosis. Proc Natl Acad Sci USA 1990; 87:3894-8. 
22. Srisawasdi P, Chaloeysup S, Teerajetgul Y, Pocathikorn A, Sukasem C, Vanavanan S. et al. Estimation of plasma small dense LDL cholesterol from classic lipid measures. Am $\mathrm{J}$ Clin Pathol 2011;136:20-9

23. Friedewald WT, Levy RI, Fredrickson DS. Estimation of the concentration of low-density lipoprotein cholesterol in plasma, without use of the preparative ultracentrifuge. Clin Chem 1972;18:499-502.

24. Abbott RD, Garrison RJ, Wilson PW, Epstein FH, Castelli WP, Feinleib M, et al. Joint distribution of lipoprotein cholesterol classes. The Framingham study. Arterioscler Thromb Vasc Biol 1983;3:260-72.

25. Millar JS, Lichtenstein AH, Cuchel M, Dolnikowski GG, Hachey DL, Cohn JS, et al. Impact of age on the metabolism of VLDL, IDL, and LDL apolipoprotein B-100 in men. J Lipid Res 1995; $36: 1155-67$.

26. Patalay M, Lofgren IE, Freake HC, Koo SI, Fernandez ML. The lowering of plasma lipids following a weight reduction program is related to increased expression of the LDL receptor and lipoprotein lipase. J Nutr 2005;135:735-9.

27. Oh SY, Miller LT. Effect of dietary egg on variability of plasma cholesterol levels and lipoprotein cholesterol. Am J Clin Nutr 1985;42:421-31.

28. Morgan AE, Mooney KM, Wilkinson SJ, Pickles NA, Mc Auley MT. Cholesterol metabolism: a review of how ageing disrupts the biological mechanisms responsible for its regulation. Ageing Res Rev 2016;27:108-24.

29. Mutungi G, Ratliff J, Puglisi M, Torres-Gonzalez M, Vaishnav U, Leite $\mathrm{JO}$, et al. Dietary cholesterol from eggs increases plasma HDL cholesterol in overweight men consuming a carbohydrate-restricted diet. J Nutr 2008;138:272-6.

30. Harman NL, Leds AR, Griffin BA. Increased dietary cholesterol does not increase plasma low density lipoprotein when accompanied by an energy-restricted diet and weight loss. Eur J Nutr 2008;47:287-93.

31. Chapman MJ, Laplaud PM, Luc G, Forgez P, Bruckert E, Goulinet S, et al. Further resolution of the low density lipoprotein spectrum in normal human plasma: physicochemical characteristics of discrete subspecies separated by density gradient ultracentrifugation J Lipid Res 1988;29:442-58.

32. Shen MM, Krauss RM, Lindgren FT, Forte TM. Heterogeneity of serum low-density lipoproteins in normal human subjects. J Lipid Res 1981;22:236-44.

33. Deckelbaum RJ, Eisenberg S, Oschry Y, Butbul E, Sharon I, Olivecrona T. Reversible modification of human plasma low density lipoproteins toward triglyceride-rich precursors. A mechanism for losing excess cholesterol esters. J Biol Chem 1982;257:6509-17.

34. Hirayama S, Miida T. Small dense LDL: an emerging risk factor for cardiovascular disease. Clin Chim Acta 2012;414: 215-24.

35. Coresh J, Kwiterovich PO. Small, dense low-density lipoprotein particles and coronary heart disease risk: a clear association with uncertain implications. JAMA 1996;276:914-5.

36. Stampfer MJ, Krauss RM, Ma J, Blanche PJ, Holl LG, Sacks FM, et al. A prospective study of triglyceride level, lowdensity lipoprotein particles diameter, and risk of myocardial infarction. JAMA 1996;276:882-8.

37. Blesso CN. Andersen CJ, Barona J, Volek JS, Fernandez ML. Whole egg consumption improves lipoprotein profiles and insulin sensitivity to a greater extent than yolk-free egg substitute in individuals with metabolic syndrome. Metabolism 2013;62:400-10.

38. Wood RJ, Volek JS, Liu Y, Shachter NS, Contois JH, Fernandez ML. Carbohydrate restriction alters lipoprotein metabolism by modifying VLDL, LDL and HDL subfraction distribution and size in overweight men. J Nutr 2006;136: 384-9.

39. Techakriengkrai T, Klangjareonchai T, Pakpeankitwattana V, Sritara $\mathrm{P}$, Roongpisuthipong C. The effect of ingestion of egg and low density lipoprotein (LDL) oxidation on serum lipid profiles in hypercholesterolemic women. Songklanakarin. J Sci Technol 2012;34:173-8.

40. DiMarco DM, Norris GH, Millar CL, Blesso CN, Fernandez ML. Intake of up to 3 eggs per day is associated with changes in HDL function and increased plasma antioxidants in healthy, young adults. J Nutr 2017;147:323-9. 